\title{
Contribución de las variables que estimulan la producción agraria en España (1990-2009)
}

\author{
SILVIA ANDRÉS GONZÁLEZ-MORALEJO \\ Grupo de Economía Internacional. Departamento de Economía y Ciencias Sociales, UNIVER- \\ SIDAD POLITÉCNICA DE VALENCIA, ESPAÑA.E-mail: silangle@upvnet.upv.es
}

\section{Mo JOSÉ SOLDADO LLORENS}

Grupo de Economía Internacional. Departamento de Economía y Ciencias Sociales, UNIVERSIDAD POLITÉCNICA DE VALENCIA, ESPAÑA.E-mail: mjsoldado@hotmail.com

\begin{abstract}
RESUMEN
El objeto del trabajo es analizar la evolución de la producción de las principales materias primas agrarias en España durante el periodo 1990-2009. Se definen las materias primas principales mediante cálculo del porcentaje del valor de la producción de cada materia prima con respecto al valor de la producción total del subsector agrario al que pertenece. Posteriormente y gracias a la ordenación descendente de estos valores, se consideran materias primas principales a las primeras de dicha ordenación en cada subsector. Se examina mediante técnicas estadísticas multivariantes la relación que existe entre la evolución de la producción de cada materia prima principal y variables tales como los precios percibidos por los productores, las reformas de la PAC o los cambios observados en otras variables relevantes como la superficie cosechada o el rendimiento. Los resultados reflejan que las variables territoriales y el precio percibido son las variables que mejor explican el Valor de la Producción Agrícola española.
\end{abstract}

Palabras clave: Materia prima principal, valor de la producción, precio, PAC.

\section{Analyse of the Main Variables Which Better Contribute to the Agricultural Production in Spain (1990-2009)}

\begin{abstract}
The aim of this paper consists on analysing the evolution of the production of major agricultural commodities in Spain during the period 1990-2009. The main raw materials are defined by calculating the percentage of the following ratio: the value of production of each raw material to the value of total production of its agriculture sub-sector. Subsequently, the decreasing order of those values lets us consider the main raw materials as the first ones of that order for each sub-sector. Then we can examine, using multivariate statistical techniques, the relation between changes in the production of each major raw material and other variables such as the prices got by farmers, the CAP reforms and the changes in other relevant variables like the area harvested or the crop yield. The results show us that those which best explain the Value of Spanish Agricultural Production are the land variables and the price received.
\end{abstract}

Keywords: Major Row Material, Value of Production, Price, CAP.

Clasificación JEL: Q11

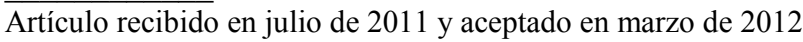

Artículo disponible en versión electrónica en la página www.revista-eea.net, ref. ə-30110 


\section{INTRODUCCIÓN}

Durante el periodo 1990-2009, la agricultura española se ha visto influida por diversos factores económicos, ecológicos y tecnológicos. Pero el rasgo fundamental es que se ha convertido en la segunda beneficiaria de la Política Agraria Común (PAC), en el marco de una creciente apertura de los mercados alimentarios instaurada con el Acuerdo sobre Agricultura de la Ronda Uruguay del GATT (Acuerdo General de Aranceles y Comercio).

Además, el trienio 2006-2008 ha supuesto una sacudida sin precedentes en los precios internacionales de las principales materias primas agroalimentarias (cereales, oleaginosas y leche). La elevación de los precios era un hecho esperado, consecuencia del crecimiento de la demanda, tanto en economías emergentes (mayor consumo per capita e incremento de la población) como en las economías desarrolladas (por la nueva demanda para la producción de biocombustibles). Sin embargo, la magnitud del incremento, cercano al $100 \%$ en muchos casos, no había sido vaticinada por ninguna de las instituciones (FAO, OCDE, USDA, Comisión Europea) que realizan ejercicios de prospectiva de mercados. Los efectos han sido especialmente patentes sobre el coste de los alimentos en los países menos desarrollados, provocando una verdadera crisis alimentaria, y también han contribuido al incremento de la inflación en las economías desarrolladas. Pero la historia se repite y el año 2010 ha concluido encaminando al mundo hacia un nuevo récord en el precio de los alimentos.

El boom de los precios de las materias primas mejora sin duda las expectativas del sector agrícola, pero una mayor demanda exige también una mayor producción. No obstante, un reciente informe del Banco Central Europeo (BCE, 2011) alerta sobre la incertidumbre con respecto a si la oferta será capaz de abastecer la creciente demanda y, en consecuencia, si se logrará limitar en el futuro el aumento de los precios de los alimentos. Esta argumentación nos conduce hacia una vía de análisis empírico, que se plasma en las siguientes preguntas de investigación: ¿la drástica subida de precios de las principales materias primas agropecuarias ha provocando un aumento de su producción?, ¿y el entramado de reformas que ha sufrido la PAC?, ¿cómo ha evolucionado dicha producción en los últimos tiempos?, ¿qué relación existe entre la evolución de la producción de cada materia prima y los cambios observados en las principales variables de las que depende?

En este contexto, el objeto del presente trabajo es analizar la evolución de la producción de las principales materias primas agropecuarias en España durante el periodo 1990-2009. Esto implica que es necesario dar respuesta a los siguientes objetivos específicos:

- Definir las materias primas principales mediante cálculo del porcentaje del valor de la producción de cada materia prima con respecto al valor de la producción total del subsector agrario al que pertenece y ordenar de 
forma descendente estos valores. Denominamos materias primas principales a las primeras materias primas de cada subsector.

- Analizar el valor de la producción de las materias primas seleccionadas como principales, con el propósito de ajustar sus curvas de tendencia durante el periodo objeto de estudio.

- Examinar, mediante técnicas estadísticas multivariantes, la relación que existe entre la evolución de la producción de cada materia prima principal, los precios percibidos por los productores y las subvenciones de la PAC, así como también con los cambios observados en otras variables relevantes de las que depende la producción como la superficie cosechada o el rendimiento.

De este trabajo se derivan importantes implicaciones para los académicos y los responsables de la toma de decisiones. Primero desvela cuáles son las principales materias primas de la agricultura española y cómo ha evolucionado su producción. Luego descubre cuáles son las variables que influyen en la producción en cada caso concreto y en qué medida lo hacen. Por último, demuestra específicamente si la producción tiene relación con aquellos hechos que han situado al sistema agroalimentario en el centro del debate económico mundial, como la reforma de la PAC o las dramáticas tensiones alcistas en las cotizaciones de dichas materias primas durante 2006-2008.

El artículo se estructura de la siguiente manera. Después de esta introducción, el segundo epígrafe repasa los antecedentes más relevantes en el estudio de la producción agropecuaria española. En el tercer epígrafe se determinan las principales materias primas y la evolución de su producción. La sección cuarta incluye los resultados más relevantes del análisis multivariante y una discusión de los mismos. Finalmente, el último epígrafe recoge las principales conclusiones del trabajo y subraya las directrices para futuras investigaciones.

\section{ANTECEDENTES}

Dos hechos consumados presiden el debate actual en torno al sistema agroalimentario, siendo el detonante de los objetivos planteados en este trabajo. De un lado, la globalización de los mercados agrarios, que ha condicionado seriamente el devenir de la PAC. Por otro, las recientes tensiones en los precios de las materias primas y su extensión al mercado de los alimentos, que entroncan con el nuevo papel de la agricultura como suministradora de energía, con graves repercusiones en un entorno de crisis económica mundial. Veámoslo.

Desde 1990 muchos acontecimientos han sucedido dentro de la PAC. A inicios de los 90, a las crisis financieras se añadía otro factor de presión: las negociaciones multilaterales de la Ronda Uruguay, que habían comenzado en 1986. Para alcanzar un acuerdo de liberalización que afectase a los productos 
agrarios, la Comunidad Europea tenía que reformar su política de precios y mercados, bajando los precios institucionales y los subsidios a la exportación. En 1992, el Consejo logró un acuerdo político para reformar la PAC (reforma MacSharry), afectando a los productos continentales mediante un sistema de ayudas directas a los productores con una cuantía fija por hectárea y por cabeza de ganado. La contribución esencial de la reforma MacSharry fue la sustitución de un modelo de protección basado en los precios por otro basado en ayudas directas, más transparente y financiado por el contribuyente. Posteriormente, la Agenda 2000 (Tangermann, 1997) profundizó en las orientaciones de 1992. Se había impulsado una filosofía de reforma, que cuestionaba por primara vez el enfoque de producción intensiva promovido por la PAC en las tres décadas anteriores.

Las reformas introducidas en 2003 y 2008, denominadas Revisión Intermedia y Chequeo Médico respectivamente ${ }^{1}$, han consolidado el sistema de Pago Único (PU) como el mecanismo más importante de apoyo a la agricultura. Los agricultores son beneficiarios de unos derechos de ayuda independientes de su producción actual. Así, el PU está sustituyendo a las intervenciones en los mercados y a los pagos directos acoplados a la producción, que predominaron a lo largo de los 80 y 90 respectivamente. Los derechos de ayuda se calculan en función de los pagos recibidos en un periodo de referencia (modelo histórico) o sobre el número de hectáreas admisibles que dispone el agricultor en el primer año de aplicación del sistema (modelo regional). España eligió el modelo histórico, por lo que la cuantía del PU se basa en las transferencias realmente percibidas por el productor en el periodo de referencia 2000-2002. Una de las principales críticas que se achaca al proceso de reforma de la PAC es que es heredero de un modelo de apoyo basado en la producción que no se ajusta a las necesidades actuales de la agricultura ni a lo que la sociedad espera de ella. Los instrumentos han cambiado pero, incluso con el PU, se ha mantenido el statu quo distributivo y presupuestario, pues la cuantía que hoy percibe el productor se deriva de criterios históricos que apenas modifican los desequilibrios tradicionales de la PAC entre explotaciones y regiones, por tanto se traslada la inercia y las desigualdades de las formas previas de apoyo a los tiempos actuales, pudiendo tener este hecho algún efecto en la evolución de la producción.

Por otra parte, en 2010 los precios de los alimentos han crecido significativamente, como sucedió en 2007 y $2008^{2}$. Parece lógico anticipar que estos hechos actúen como estimulo de la producción. Instituciones como el Banco Central

\footnotetext{
${ }^{1}$ Los principios de la Revisión Intermedia y del Chequeo Médico de la PAC, y sus consecuencias, se encuentran detalladamente explicados en los libros de García (2006) y García y GómezLimón (2010).

${ }^{2}$ Headey y Fan (2010) ofrecen un extenso informe que reflexiona sobre las causas de la crisis alimentaria global de 2008, explicando qué ocurrió y cómo lograr su prevención en el futuro.
} 
Europeo (BCE, 2011) o la Organización de las Naciones Unidas para la Agricultura y la Alimentación (FAO, 2011) han mostrado su inquietud, especialmente por la contribución de factores coyunturales como estructurales que presionarán sobre la tendencia al alza a medio y largo plazo. El principal motivo coyuntural radica en las malas condiciones climatológicas sufridas por los grandes productores en 2010 y que han mermado sus cosechas. Incluso el rebrote proteccionista de algunos países y los fondos de inversión que se retiraron del mercado de materias primas como respuesta a la crisis mundial, y que ahora retornan buscando más rendimiento, pueden haber contribuido. Pero los factores que generan más inquietud son los estructurales. La creciente demanda de los países emergentes, encabezados por China e India, donde el incremento de los ingresos de la población modifica sus hábitos alimenticios, promoviendo el consumo de productos de mayor valor añadido como carnes y lácteos (incrementando a su vez la demanda de cereales y oleaginosas destinadas a la alimentación animal). Otro factor estructural es que en algunas materias primas (azúcar y cereales) el uso alimentario se enfrenta a la intensa competencia para la producción de biocombustibles ${ }^{3}$.

Figura 1

Índice de la FAO para los precios de los alimentos

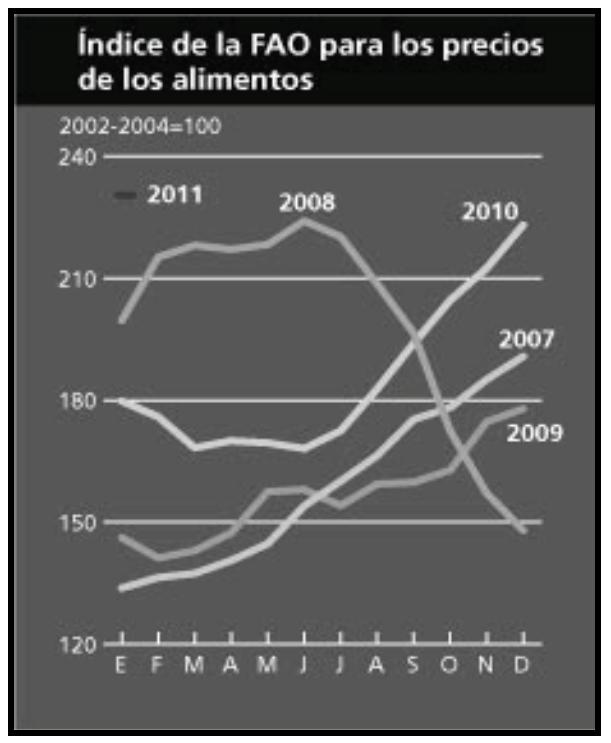

Fuente: FAO (2011b).

\footnotetext{
${ }^{3}$ La contribución de los biocombustibles a la elevación del precio de las materias primas resulta controvertida en la literatura. Para profundizar sobre esta cuestión y sobre la volatilidad del mercado de las materias primas véase Atance y García (2008).
} 
El índice elaborado por la FAO mostrado en la Figura 1, que incluye la media ponderada de los precios de exportación de carnes, lácteos, cereales, aceites, grasas y azúcar, alcanzó en junio de 2008 los 213 puntos, mientras que en diciembre de 2010 ya rozaba los 215, con productos como el azúcar, el aceite y las grasas en los que se duplica en tan sólo dos años. La FAO se apoya en estos datos para avalar la peligrosidad de la situación, puesto que será duradera y todavía existe margen para que los precios continúen creciendo algo más.

A lo largo del periodo objeto de estudio se han publicado numerosos trabajos que, utilizando diferentes aproximaciones, han tratado el análisis de la productividad agraria y las funciones de producción agregadas. Respecto a las tendencias y factores explicativos de la productividad agraria se puede encontrar un gran número de aportaciones centradas en los resultados y tendencias a escala nacional; véanse por ejemplo, Gutiérrez (2000), Coelli y Rao (2005) y Rezitis (2005). Y aunque menores, debido probablemente a la ausencia de datos estadísticos al nivel de desagregación requerido, también existen aportaciones que analizan las tendencias de las agriculturas regionales, destacando en el caso europeo los trabajos de Castillo y Cuevas (2006) y Ezcurra et al. (2008). Respecto a las funciones de producción, es abundante su elaboración en el ámbito agrario, pudiendo destacarse como ejemplo de funciones a nivel micro el trabajo de Cañas et al. (1994), referido específicamente al caso del maíz, y a nivel del sector agrícola en su conjunto el de Cepas y Dios (1999), que estima la función de producción agregada para el sector agrario español con el valor añadido de que evalúa la incidencia de la multicolinealidad para distintos niveles de agregación. El presente trabajo concentra su atención en el planteamiento de funciones de oferta para diferentes materias primas agrícolas (enfoque sectorial por productos), por lo que se diferencia especialmente de los artículos anteriormente comentados en el tipo de variables consideradas, a la vez que ensaya la introducción de variables dummies para analizar el efecto en la producción de los últimos acontecimientos a los que aludíamos al comienzo del artículo. En el caso que nos ocupa, el planteamiento de la curva de oferta de una materia prima agraria ofrece dos utilidades fundamentales: por un lado, nos permite conocer cómo afectan los diferentes determinantes ensayados a su producción total, y por otro, nos permite realizar predicciones sobre la evolución futura de la producción.

\section{IDENTIFICACIÓN DE LAS MATERIAS PRIMAS PRINCIPALES}

En la selección de las principales materias primas agropecuarias de la agricultura española se ha tomado como fuente de información el Anuario de Estadística Agroalimentaria, publicado por el Ministerio de Agricultura. El Anuario define 18 grupos agropecuarios; se ha decidido la eliminación de 5 de ellos, 
cultivos forrajeros, flores y plantas ornamentales, otros cultivos leñosos, lana y pieles y miel y cera, debido a su escaso interés alimentario. La Tabla 1 recoge los grupos que se han clasificado inicialmente para este trabajo, así como los cultivos o productos principales que los componen ${ }^{4}$.

Tabla 1

Grupos productivos agropecuarios de interés alimentario

\begin{tabular}{ll}
\hline \multicolumn{1}{c}{ GRUPOS PRODUCTIVOS } & \multicolumn{1}{c}{ PRODUCTOS PRINCIPALES } \\
\hline Cereales grano & Trigo, cebada, avena, centeno, triticale, arroz, maíz, sorgo \\
\hline Leguminosas grano & $\begin{array}{l}\text { Judías secas, habas secas, lentejas, garbanzos, guisantes secos, veza, yeros, } \\
\text { altramuz }\end{array}$ \\
\hline Tubérculos consumo humano & Patata \\
\hline Cultivos industriales & Caña de azúcar, remolacha azucarera, girasol, soja \\
\hline Hortalizas & $\begin{array}{l}\text { Col, espárrago, lechuga, escarola, espinaca, acelga, sandía, melón, pepino, } \\
\text { calabacín, berenjena, tomate, pimiento, fresa, alcachofa, coliflor, ajo, cebolla, } \\
\text { zanahoria, champiñón, judías verdes, guisantes verdes, habas verdes }\end{array}$ \\
\hline Cítricos & Naranja, mandarina, limonero, pomelo \\
\hline Frutales fruto fresco no cítricos & $\begin{array}{l}\text { Manzana, pera, níspero, albaricoque, cereza, melocotón, ciruela, higo, } \\
\text { chirimoya, aguacate, plátano, kiwi, granada }\end{array}$ \\
\hline Frutales fruto seco & Almendra, avellana, nuez \\
\hline Viñedo & Uva dedicada al consumo en fresco \\
\hline Olivar & Aceituna para aderezo \\
\hline Carne & Bovino, ovino, caprino, porcino, equino, aves, conejo \\
\hline Leche & Leche de vaca, leche de oveja, leche de cabra \\
\hline Huevos & Huevos \\
\hline
\end{tabular}

Fuente: Elaboración propia a partir del Anuario de Estadística Agroalimentaria (2009).

Dado que el número de grupos productivos destinados al consumo humano resulta elevado para los objetivos del trabajo, de los grupos que aparecen en la Tabla 1 se han seleccionado aquellos cuyo porcentaje con respecto al valor de la Producción de la Rama Agraria Nacional desde 1990 al 2009 sea igual o superior al $3 \%$, porcentaje cuyo uso permite descartar los menos significativos a nivel nacional. Para realizar este ejercicio se ha calculado el valor total de la

\footnotetext{
${ }^{4}$ Respecto a los capítulos correspondientes al viñedo y al olivar hay que indicar que sólo se han considerado como productos principales la uva dedicada al consumo en fresco y la aceituna para aderezo. Esto es debido a dos razones fundamentales. En primer lugar, aunque la producción de vino y aceite es especialmente relevante en España, quedan excluidas de nuestro análisis empírico ya que se trata de productos transformados. En segundo lugar, las diferentes calidades de los productos destinados a la transformación provoca la existencia de múltiples precios percibidos por los agricultores, motivo por el cual el Anuario no ofrece un valor de la producción referido al total de uva o aceitunas producidas.
} 
producción (VTP) de cada grupo en el periodo 1990-2009 y se ha obtenido el porcentaje que representa sobre el Valor Total de la Producción de la Rama Agraria Nacional (VTPRA) en el mismo periodo ${ }^{5}$. Los resultados obtenidos se recogen en la Tabla 2, que muestra los grupos que más aportan al VTPRA, y que serán incluidos en el análisis empírico: carnes, hortalizas, cereales grano, leche, frutales de fruto fresco no cítrico y cítricos.

Tabla 2

Contribución porcentual de los grupos productivos al Valor Total de la Producción de la Rama Agraria Nacional

\begin{tabular}{lcc}
\hline \multicolumn{1}{c}{ GRUPOS PRODUCTIVOS } & $\begin{array}{c}\text { VPT (1990-2009) } \\
\text { Millones de euros }\end{array}$ & $\begin{array}{c}\text { Porcentaje sobre el } \\
\text { VTPRA nacional }\end{array}$ \\
\hline Carne & 157.508 & $26,22 \%$ \\
\hline Hortalizas & 84.743 & $14,11 \%$ \\
\hline Cereales grano & 48.792 & $8,12 \%$ \\
\hline Leche & 38.830 & $6,46 \%$ \\
\hline Frutales fruto fresco no cítrico & 26.158 & $4,35 \%$ \\
\hline Cítricos & 20.454 & $3,41 \%$ \\
\hline Huevos & 13.418 & $2,23 \%$ \\
\hline Tubérculos consumo humano & 10.761 & $1,79 \%$ \\
\hline Cultivos industriales & 10.657 & $1,77 \%$ \\
\hline Frutales fruto seco & 4.366 & $0,73 \%$ \\
\hline Olivar & 3.482 & $0,58 \%$ \\
\hline Viñedo & 2.591 & $0,43 \%$ \\
\hline Leguminosas grano & 2.154 & $0,36 \%$ \\
\hline VPRA nacional & $\mathbf{6 0 0 . 6 6 6 , 2 0}$ & $\mathbf{1 0 0 \%}$ \\
\hline
\end{tabular}

Fuente: Elaboración propia a partir del Anuario de Estadística Agroalimentaria (2009).

Para los grupos seleccionados en la Tabla 2 se define qué cultivos o productos dentro del grupo son los que más valor aportan. Para ello se ha calculado el porcentaje del valor de la producción de cada producto con respecto al valor total de la producción del grupo correspondiente. Se denominarán materias pri-

${ }^{5}$ Considerando que se tiene un número " $\mathrm{n}$ " de años, " $\mathrm{k}$ " productos en el grupo " $\mathrm{r}$ " $\mathrm{y}$ " $\mathrm{t}$ " grupos productivos, el detalle algebraico será:

$$
V T P_{j}=\sum_{i=1}^{n} V p(r) ; \quad V T P_{r}=\sum_{j=1}^{k} V T \not(r)
$$

De donde:

Vpi: Valor de la producción del producto $j$ en el año i.

VTPj: Valor total de la producción del producto $j$ en el periodo 1990-2009.

VTPr: Valor total de la producción del grupo $r$ en el periodo 1990-2009. 
mas principales a aquellas que ordenadas de forma descendiente sumen una cantidad igual o superior al $40 \%{ }^{6}$ del valor total de la producción del grupo durante el periodo objeto de estudio ${ }^{7}$.

Aplicando la fórmula anterior se obtienen las materias primas principales dentro de cada grupo agropecuario. Los resultados se recogen en la Tabla 3.

Se han identificado 8 materias primas principales. En los cereales, el cultivo más importante a resaltar es la cebada que muestra un peso del 41,65 por ciento; las hortalizas tomate y pimiento destacan con valores respectivos del 27,3 y 12,2 por ciento; la naranja contribuye con un 43,85 por ciento a los cítricos; melocotón y pera participan con pesos respectivos del 31 y 14,96 por ciento en el grupo de frutales de fruto fresco no cítrico; en ganadería es indudable el liderazgo de la producción de porcino dentro de las carnes, siendo su aportación prácticamente del 40 por ciento, y de la leche de vaca en el subsector lácteo con un peso del 79,11 por ciento. Definidas las materias primas principales se han ajustado sus curvas de tendencia temporales a lo largo del periodo 1990-2009, mediante mínimos cuadrados. Se ha encontrado que todas muestran una tendencia ascendente en su cantidad producida durante el periodo de estudio, aunque se observan dos tipos diferenciados de crecimiento. En el caso del tomate, el pimiento y el porcino, la tendencia de crecimiento es sostenida y estable en el

\footnotetext{
${ }^{6}$ En la literatura disponible se utilizan distintos porcentajes para la definición de materia prima principal. El uso de porcentajes más restrictivos es común cuando el análisis incluye regiones de muy distinta especialización productiva, como sucede en España, por lo que conviene reducir el número de cultivos y producciones ganaderas que supera el porcentaje designado. Véase en Mora y Rojas (2007) el ejemplo de una agricultura menos diversificada en la que se apuesta por establecer un porcentaje menos restrictivo.

${ }^{7}$ Considerando que se tiene un número " $\mathrm{n}$ " de años, "k" productos en el grupo " $\mathrm{r}$ " y " $\mathrm{t}$ " grupos productivos, la siguiente expresión resume el concepto de materia prima principal (MPP):
}

$$
M P P=\sum_{j=1}^{k} \operatorname{VTPj}(r) \geq 0,40
$$

De donde:

$$
\%_{V T P_{j}}=\frac{V T P_{j}}{V T \mathrm{P}_{\mathrm{r}}}=\frac{\sum_{i=1}^{n} V P_{i}(r)}{\sum_{j=1}^{k} V T P_{j}(r)}=\frac{\sum_{i=1}^{n} V P_{i}(r)}{\sum_{j=1}^{k} \sum_{i=1}^{n} V P_{i}(r)}
$$

Entendiéndose por:

\%VTPj: Porcentaje del Valor Total de la Producción del producto $j$ en el grupo $r$.

Vpi: Valor de la producción del producto $j$ en el año $i$.

VTP $j$ : Valor total de la producción del producto $j$ en el periodo 1990-2009. 
tiempo y los coeficientes de determinación del ajuste más altos. En el caso de cebada, naranja, pera, melocotón y leche de vaca, la tendencia de crecimiento es más inestable y presenta altibajos.

Tabla 3

Materias primas principales

\begin{tabular}{|c|c|c|c|c|c|}
\hline $\begin{array}{c}\text { GRUPOS } \\
\text { PRODUCTIVOS }\end{array}$ & $\begin{array}{l}\text { PRODUCTOS } \\
\text { PRINCIPALES }\end{array}$ & $\%$ VTPj & $\begin{array}{c}\text { GRUPOS } \\
\text { PRODUCTIVOS }\end{array}$ & $\begin{array}{l}\text { PRODUCTOS } \\
\text { PRINCIPALES }\end{array}$ & $\%$ VTPj \\
\hline \multirow{8}{*}{ Cereales grano } & Cebada & $41,65 \%$ & \multirow{4}{*}{ Cítricos } & Naranjo & $43,85 \%$ \\
\hline & Trigo & $29,99 \%$ & & Mandarino & $38,91 \%$ \\
\hline & Maíz & $20,19 \%$ & & Limonero & $16,80 \%$ \\
\hline & Arroz & $6,97 \%$ & & Pomelo & $0,46 \%$ \\
\hline & Avena & $3,26 \%$ & \multirow{13}{*}{$\begin{array}{c}\text { Frutales fruto fresco } \\
\text { no cítricos }\end{array}$} & Melocotón & $31,00 \%$ \\
\hline & Centeno & $1,02 \%$ & & Pera & $14,96 \%$ \\
\hline & Triticale & $0,33 \%$ & & Manzana & $14,80 \%$ \\
\hline & Sorgo & $0,24 \%$ & & Plátano & $8,82 \%$ \\
\hline \multirow{23}{*}{ Hortalizas } & Tomate & $27,30 \%$ & & Cereza & $8,04 \%$ \\
\hline & Pimiento & $12,20 \%$ & & Ciruela & $5,26 \%$ \\
\hline & Judias verdes & $6,61 \%$ & & Albaricoque & $4,20 \%$ \\
\hline & Lechuga & $6,54 \%$ & & Aguacate & $3,92 \%$ \\
\hline & Melón & $5,78 \%$ & & Higo & $3,50 \%$ \\
\hline & Fresa y Fresón & $5,60 \%$ & & Níspero & $2,21 \%$ \\
\hline & Ajo & $4,04 \%$ & & Chirimoya & $1,71 \%$ \\
\hline & Pepino & $3,81 \%$ & & Granada & $0,81 \%$ \\
\hline & Cebolla & $3,16 \%$ & & Kiwi & $0,59 \%$ \\
\hline & Sandía & $2,85 \%$ & \multirow{7}{*}{ Carne } & Porcino & $39,92 \%$ \\
\hline & Coliflor & $2,84 \%$ & & Bovino & $24,18 \%$ \\
\hline & Alcachofa & $2,82 \%$ & & Ovino & \multirow{2}{*}{$17,45 \%$} \\
\hline & Calabacín & $2,35 \%$ & & Caprino & \\
\hline & Espárrago & $2,27 \%$ & & Aves & $14,27 \%$ \\
\hline & Champiñón & $1,75 \%$ & & Conejo & $3,36 \%$ \\
\hline & Col & $1,68 \%$ & & Equino & $0,81 \%$ \\
\hline & Zanahoria & $1,56 \%$ & \multirow{3}{*}{ Leche } & Leche de vaca & $79,11 \%$ \\
\hline & Berenjena & $1,41 \%$ & & Leche de oveja & $12,45 \%$ \\
\hline & Habas & $0,96 \%$ & & Leche de cabra & $8,45 \%$ \\
\hline & Guisantes & $0,83 \%$ & & & \\
\hline & Escarola & $0,54 \%$ & & & \\
\hline & Espinaca & $0,53 \%$ & & & \\
\hline & Acelga & $0,45 \%$ & & & \\
\hline
\end{tabular}

Fuente: Elaboración propia a partir del Anuario de Estadística Agroalimentaria (2009). 


\section{DETERMINANTES RELEVANTES EN LA PRODUCCIÓN DE LAS MATERIAS PRIMAS PRINCIPALES: CONTRASTE EMPÍRICO}

\subsection{Descripción de los datos y método de análisis}

\section{Selección de las variables dependientes y explicativas}

El comportamiento del agricultor es un sistema complejo donde está en permanente interacción con su entorno socioeconómico y físico. El agricultor, a la hora de tomar decisiones, tiene en cuenta consideraciones relacionadas con su entorno económico, social, cultural y ambiental. Según Reig y Picazo (2002), en la oferta de un producto agrícola inciden múltiples factores, tales como el precio del producto, los precios de los productos con los que guarda relación en la esfera productiva, el acceso y precio de los factores de producción, los cambios en la tecnología, la precipitación pluvial, la información, etc. Respecto a su incidencia en el tiempo, algunos factores como los precios de productos e insumos y la precipitación pluvial, tienen vigencia en todos los horizontes de planeación del productor. Los factores institucionales influyen en el medio y largo plazo, destacando los cambios en el acceso a la tierra y los factores ecológicos que influyen en el largo plazo. Sin embargo, algunos constituyen un conjunto de factores no modificables o con poca posibilidad de manejo, como las características climáticas, la topografía y las cualidades del suelo.

En base a lo anterior, hemos construido las siguientes variables dependientes y explicativas. Como dependientes hemos creado las variables $V T P_{j}$, que representan el Valor Total de la Producción de cada materia prima principal en los años del periodo 1990-2009, en miles de euros constantes. Para transformar a precios constantes se ha utilizado como deflactor el Índice de Precios Percibidos por los Productores, suponiendo como año base el año 2000. Los datos requeridos para la obtención de las variables dependientes han sido extraídos del Anuario de Estadística Agroalimentaria. Con esta información, se procedió a estimar un conjunto de modelos donde el Valor Total de la Producción de cada materia prima es explicado por las variables independientes que definimos a continuación (la Tabla 4 resume las variables dependientes y explicativas y su influencia esperada):

Variables territoriales: superficie y rendimiento. Diversos autores sostienen que los factores territoriales son determinantes en la agricultura ya que, salvo en empresas ganaderas, la superficie disponible y los rendimientos de las explotaciones condicionan su dimensión económica y su eficiencia (Infante, 2008; Connor y Loomis, 2002; Roberts, 2002). España está definida por el carácter dual de sus explotaciones. Dos tercios son pequeñas explotaciones de reducida dimensión, mientras que sólo un 10 por ciento de las unidades productivas absorbe casi la mitad de la superficie utilizada y la 
producción agraria obtenida. Como destacan los autores señalados existe una relación directa entre superficie disponible y producto generado. Por otra parte, Connor y Loomis (2002) alegan que la producción de una explotación es el producto matemático de la superficie cosechada y el rendimiento por unidad de superficie. El rendimiento se encuentra directamente afectado por los factores relacionados con el clima (brevedad de la estación, pluviometría...), por el carácter intensivo o extensivo de la explotación y por el cambio tecnológico (Roberts, 2002). Debido a que la Producción Final Agraria se define como multiplicación del precio por el rendimiento y por la superficie total, para evitar elevados grados de multicolinealidad se han construido las variables independientes SR, superficie en regadío dedicada al cultivo en miles de hectáreas, SS, superficie en secano dedicada al cultivo en miles de hectáreas y PP, precipitación total anual en millones de $\mathrm{m}^{3}$, como aproximación de las variables territoriales. Para las materias primas principales de origen ganadero se han considerado diferentes variables explicativas que aproximan la cabaña ganadera y sus rendimientos. Se trata de las variables QP, efectivos ganaderos de porcino en España en unidades, AS, número de animales sacrificados en miles, $\mathrm{PC}$, peso canal medio del porcino en kilos, VO, número de vacas de ordeño en miles, y RV, rendimientos obtenidos en las vacas de ordeño en litros/año. Todas las variables se han extraído del Anuario de Estadística Agroalimentaria.

Los precios. La curva de oferta es una relación directa entre cantidad ofrecida y precio del producto (o expectativas de precios o precios esperados). Por su parte, los precios de los productos competitivos pueden tener un efecto inverso, reduciéndose la oferta si las previsiones en el precio del competitivo fuesen mejores. En este trabajo entendemos por producto competitivo aquel cuya producción podría sustituir de forma ágil a la de alguna de las materias primas principales. Con el fin de incluir estas orientaciones en el modelo hemos creado las variables VIP, variación interanual del precio medio percibido por los agricultores en $€ / 100$ kilos (como aproximación del precio para evitar la correlación con la variable dependiente antes aludida), y PS, precio medio percibido por los agricultores en el caso del producto sustitutivo en $€ / 100$ kilos. La variable PS se ha considerado únicamente donde las posibilidades de sustitución son más evidentes, es decir, en los subsectores cerealícola y hortícola. Los datos sobre precios proceden del Anuario de Estadística Agroalimentaria.

Las subvenciones procedentes de la PAC. El campo español ha percibido un sustancioso volumen de fondos desde su incorporación a la actual UE, alcanzando su máximo en 2003 (Andrés y García, 2009). Ha sido ampliamente cuestionado el hecho de que los agricultores pudieran tomar sus decisiones de producción en función de la subvención recibida y no como parte de un planteamiento estratégico de futuro (García y Ruesga, 2007). Para determi- 
nar en qué grado tienen efecto las subvenciones de origen comunitario al sector hemos incluido la variable TP, que se compone a partir de las transferencias directas ${ }^{8}$ procedentes del FEOGA-GARANTÍA (FEAGA desde 1 de enero de 2007) en miles de euros constantes $(2000=100)$. Las transferencias directas se encuentran cuantificadas en los Informes sobre la Financiación de la PAC en España del FEGA (Fondo Español de Garantía Agraria). En los casos en los que el Informe refiere la ayuda directa destinada a un grupo productivo, ésta se ha repartido en función del porcentaje que cada materia prima representa dentro de su grupo.

\section{Tabla 4}

Resumen de las variables independientes y signo esperado de su influencia sobre la variable dependiente

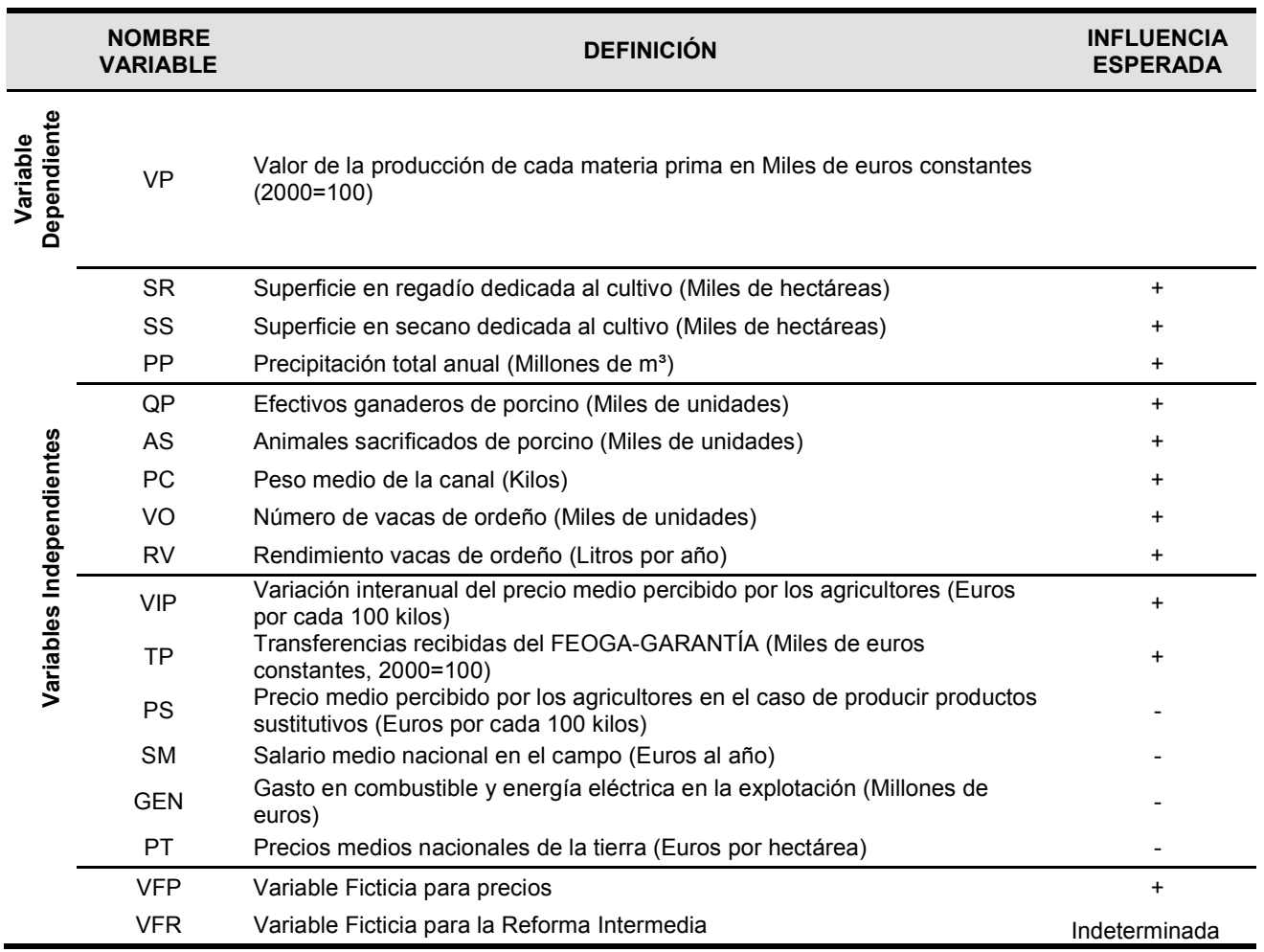

Fuente: Elaboración propia.

\footnotetext{
${ }^{8}$ En este sentido debemos matizar que las intervenciones en el mercado, las ayudas al desarrollo rural y las ayudas a la transformación no han sido consideradas en este trabajo, al no ser recibidas directamente por el agricultor.
} 
El coste de los factores de producción. Los precios de los factores productivos ejercen un efecto inverso sobre la oferta, de manera que una reducción de los costes aumentará la rentabilidad de la producción, animando a las explotaciones a incrementar su cantidad producida. Para mantener coherencia con las fuentes de información, se han elegido del Anuario de Estadística Agroalimentaria aquellas variables con capacidad para aproximar el precio de los principales factores. A partir de ellas se han definido las siguientes variables independientes: SM, salario medio nacional en el campo en euros/año; GEN, gasto en combustible y energía eléctrica en la explotación en millones de euros; PT, precios medios nacionales de la tierra en euros/ha.

Variables ficticias. La drástica subida de precios de las materias primas agroalimentarias y las recientes reformas de la PAC presiden la actualidad en el sector agroalimentario. Para contrastar las hipótesis vinculadas a ellas se han creado dos variables ficticias, VFP y VFR. La VFP es la variable ficticia diseñada para evaluar si la producción ha aumentado más desde que se dispararon los precios, por lo que toma valor 1 en los años 2007, 2008 y 2009. Por otra parte, en el pasado la PAC ha introducido severas restricciones sobre la producción agroalimentaria, que han tendido a eliminarse a medida que los mercados funcionan de manera más libre y son más flexibles las decisiones de los agricultores, lo que ha sido especialmente evidente tras la Revisión Intermedia (Atance y García, 2008). En consecuencia, la VFR es la variable ficticia diseñada para valorar la incidencia de la Revisión Intermedia, que comienza a aplicarse en España en 2006, por ello la variable toma valor 1 desde dicho año ${ }^{9}$.

\section{Análisis estadístico realizado}

Con el fin de llegar a la estimación del modelo que mejor nos permita conocer el proceso de oferta agraria, se estudian cuatro especificaciones. A continuación se describen las distintas especificaciones del modelo de regresión múltiple estimado para cada materia prima. La primera especificación se corresponde con un análisis genérico en el que se han utilizado los datos originales recogidos en las fuentes de información:

$$
\mathrm{VP}=\beta_{0}+\beta_{\mathrm{i}}^{*} \mathrm{X}_{\mathrm{i}}
$$

De donde:

$\mathrm{B}_{0}$ es el promedio del valor de la variable explicada cuando todas las variables valen 0 .

$\mathrm{B}_{\mathrm{i}}$ es el incremento del promedio de la variable explicada (VP) cuando se produce un incremento unitario en cualquiera de las variables explicativas $\left(\mathrm{X}_{\mathrm{i}}\right)$.

\footnotetext{
${ }^{9}$ Su profundización, el Chequeo Médico, comienza su aplicación en España en 2010, por lo que sus efectos quedan fuera del ámbito de este trabajo.
} 
Una vez obtenido éste se ha observado que algún dato puede no ajustarse a una distribución normal, por lo tanto, se han realizado las transformaciones convenientes para intentar aproximarlos a una distribución normal. Estas transformaciones implican el uso de logaritmos en base 10 y como resultado se obtiene el modelo logarítmico que será la segunda especificación en nuestro estudio. Puede darse el caso de que los agricultores se fijen en los datos del año anterior para tomar las decisiones de producción más apropiadas para el año actual, por lo tanto se ha ensayado un análisis en el que las variables de carácter económico son retardadas un año. La tercera especificación es el modelo con retardos. Por último, como es objetivo fundamental de este trabajo analizar el efecto de los cambios drásticos acontecidos en el precio de las materias primas y de la Reforma Intermedia de la PAC en el comportamiento de los agricultores, se ha decidido incluir 2 variables ficticias que midan dicho efecto. La cuarta especificación es el modelo con variables ficticias.

\subsection{Resultados}

La Tabla 5 recoge los resultados de las distintas especificaciones estimadas, exceptuando la especificación genérica, que ha sido omitida, salvo en el caso de la cebada, porque no asegura los supuestos básicos de normalidad y homocedasticidad y ofrece menores coeficientes de determinación. Los parámetros que estima el método de regresión múltiple son "coeficientes condicionados", por lo que debe evitarse la presencia de variables independientes altamente correlacionadas. Por ejemplo, para todas las materias primas analizadas se observa una fuerte y lógica relación entre las variables que aproximan el precio de los principales factores productivos, SM, GEN y PT, por lo que en cada estimación sólo se incluye una de ellas, concretamente la que mayor correlación muestra con la variable dependiente (este es el criterio seguido en todos los casos en los que ha sido hallada alta correlación entre variables independientes). Esta misma es la razón que aconseja que las variables ficticias se prueben por separado en cada especificación. Las especificaciones se presentan incluyendo sólo aquellas variables en las que se han obtenido parámetros significativos en las distintas pruebas efectuadas.

En el caso de la cebada, los resultados muestran que los cambios observados en el precio del producto son los únicos que contribuyen en alguna proporción a explicar su dinámica productiva. Obsérvese que en la especificación genérica del modelo el coeficiente estimado para la variable PS, única variable significativa, no presenta el signo esperado, por lo que debe considerarse que el precio del sustitutivo está actuando aquí como variable proxy del precio del propio producto (puesto que las cotizaciones de los distintos tipos de cereal suelen evolucionar en el mismo sentido). 
Tabla 5

Factores determinantes de la evolución de la producción

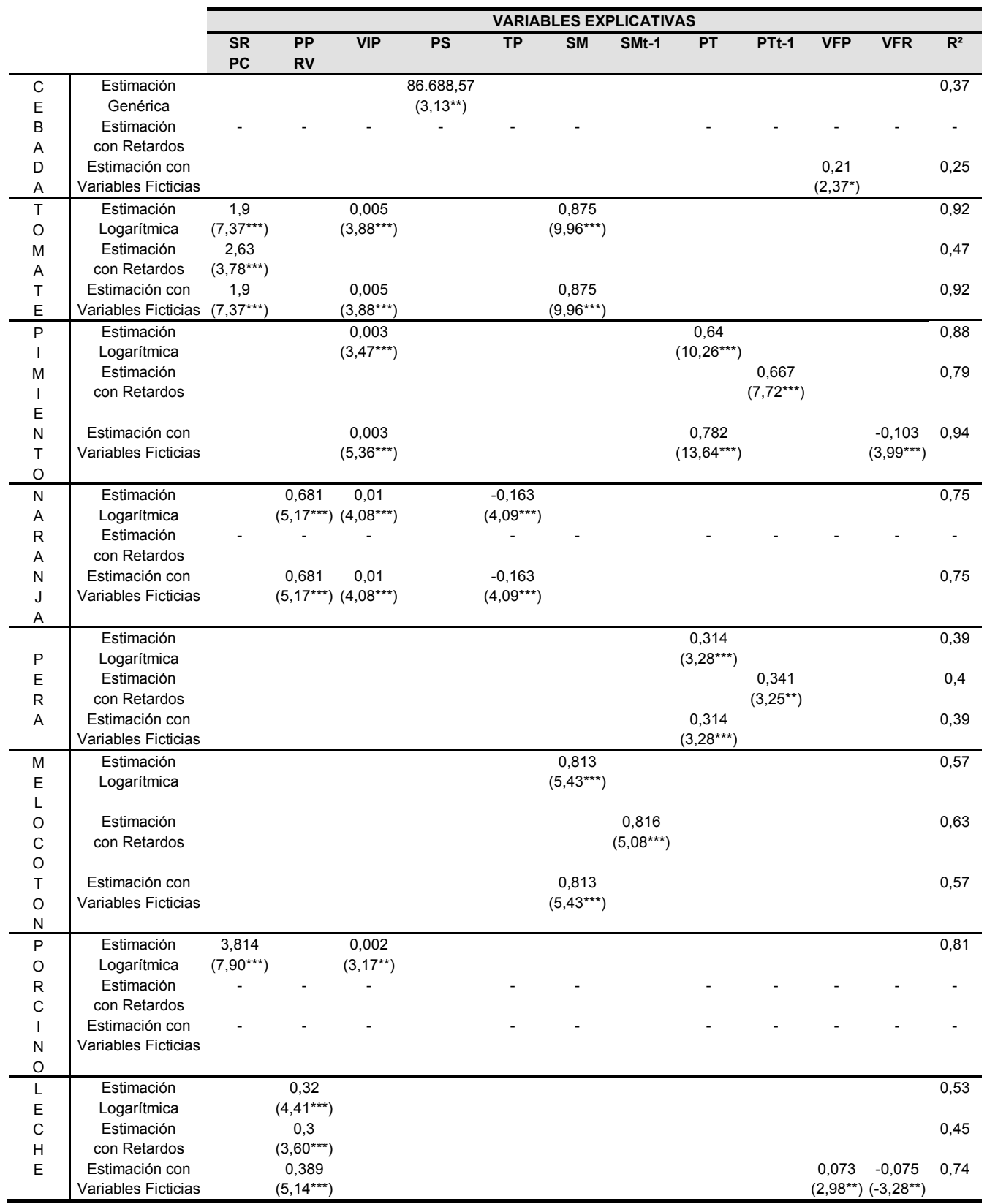

Fuente: Elaboración propia. 
Por otra parte, en la especificación con variables ficticias ha resultado significativa, tal y como era de esperar, la variable construida para verificar la incidencia positiva del alza inesperada en los precios internacionales de las principales materias primas agroalimentarias, lo que pone de manifiesto que el crecimiento de la producción de cebada en los años 2007, 2008 y 2009 se situó por encima del promedio alcanzado en los años anteriores (concretamente un 21 por ciento más). Al no hallarse parámetros significativos en la especificación con retardos se desprende que los precios de la campaña anterior no son los influyentes. Un último aspecto destacable en el ámbito cerealícola es que no han manifestado significación alguna las transferencias directas procedentes de la PAC, lo que refleja que el crecimiento de la producción de cebada en España ya no se relaciona con el volumen de las transferencias al sector, al haberse conseguido el desacoplamiento que comienza a introducirse con la Reforma de 1992.

En el subsector hortícola se detectan ciertos matices diferenciadores según el producto considerado. En el caso del tomate, los resultados respaldan la hipótesis de que la variable que mayor influencia ejerce sobre la evolución de la producción son los cambios observados en la superficie cosechada. El precio esperado de la cosecha (y no el del año anterior) también está presente en las estimaciones, aunque es mucho menor su influencia como variable explicativa. La evolución de los precios de los factores productivos es el último predictor, con un poder explicativo comprendido entre las variables SR y VIP; sin embargo su signo es positivo, contrario al esperado, lo que nos conduce a pensar que el inexorable aumento de dichos precios a largo plazo, aunque ejerza un estímulo al abandono de la producción, no lo hace con tanta intensidad como para quedar reflejado en el modelo. Obsérvese que no son significativas las transferencias directas de la PAC, pues frutas y hortalizas ha sido tradicionalmente uno de los sectores agrícolas menos subvencionado desde dicha política (las subvenciones al subsector están separadas de su nivel de producción). Tampoco aporta novedades la especificación con variables ficticias, debido a que ni el aumento de los precios de los alimentos de los últimos años ni la aplicación de la Reforma Intermedia han tenido influencia positiva alguna en el subsector hortícola. Si atendemos a las especificaciones referidas al pimiento se corroboran los principales hechos ya caracterizados para este subsector. La evolución del precio de los factores productivos (también con retardo de un año) y del precio del producto son las variables que explican la producción de pimiento, destacando el hecho de que las variables territoriales han perdido completamente su poder explicativo. El precio de los factores productivos se mantiene con signo positivo, confirmando que la dinámica productiva del subsector enmascara el posible abandono de la producción a largo plazo. Por último, ha resultado significativa la variable ficticia construida para verificar la incidencia de la Reforma Intermedia, pero su signo es negativo, lo que denota 
que el aumento medio de la producción de pimiento ha sido en promedio un 10 por ciento menor en los años de puesta en marcha de la misma.

Como interpretación de los datos referidos a la citricultura cabe concluir que los cambios observados en los rendimientos, el precio del producto y las transferencias directas de la PAC son los que contribuyen en una mayor proporción a explicar los comportamientos en la producción de naranjas. Al tratarse de un árbol frutal, dentro de las variables territoriales son los cambios observados en los rendimientos los que adquieren importancia como principal predictor, al contrario de lo que ocurre en el caso de las hortalizas. Junto al rendimiento, la variable TP se perfila aquí como otra variable explicativa relevante y presente en todos los modelos ensayados; pero obsérvese su signo negativo, que ratifica sin confusión que el aumento de las transferencias al producto no ha intensificado su producción sino todo lo contrario. Aunque con menor relevancia que las anteriores aparece también como variable explicativa el precio percibido por el agricultor.

Los precios de los factores productivos no han mostrado el efecto esperado sobre la producción de naranjas, que al tratarse de un frutal se caracteriza por la poca flexibilidad a la que se enfrenta el productor en el sentido de menor capacidad de sustitución de su producción por otra (menor movilidad de los factores productivos); aunque este hecho viene corroborado por el modelo con retardos, donde ninguna variable resultó significativa. Finalmente, parece lógico que las variables ficticias hayan resultado en este caso no significativas, pues la naranja es un producto cuyo precio más bien ha tendido a la baja durante el periodo 2007-2009, a la vez que no se ha visto afectado por el pago único durante los primeros años de aplicación de la Reforma Intermedia.

En relación con los frutales no cítricos, las estimaciones obtenidas arrojan resultados coincidentes para el peral y el melocotonero. Se confirma que ambas producciones vienen únicamente influenciadas por el precio de los factores productivos, pero lo que más sorprende es su signo positivo, que nos hace pensar que su impacto en la reestructuración de la producción no es lo suficiente intenso para reflejarse como tal en el modelo. Es la limitada capacidad de respuesta del fruticultor respecto a la sustituibilidad de factores productivos y bienes de un año a otro lo que explica esta situación. Contrariamente a lo esperado por su condición de frutales, los rendimientos y los precios del producto no han mostrado significación en el modelo. La variable TP, también sin parámetros significativos, desvincula la producción de las transferencias directas recibidas, que como es sabido siempre fueron especialmente reducidas en un sector como el hortofrutícola. Por último, obsérvese que las variables ficticias tampoco aportan novedad alguna, pues no se perciben cambios objeto de men-ción por efecto del aumento de los precios en el periodo 2007-2009 ni por efecto de la Reforma Intermedia (que no afectaba a este tipo de productos). 
Veamos los resultados obtenidos en el sector cárnico. En las especificaciones para el porcino la principal variable explicativa es el peso de la canal seguida del precio del producto, ambas con el signo esperado. Parece razonable que en el modelo con retardos ninguna variable sea significativa, pues la producción cárnica adolece de una problemática similar a lo ya comentado en el caso de los árboles frutales (escasa capacidad de reacción de los productores de un año a otro). Quizá esta misma razón sea la que explica por qué las variables ficticias tampoco han resultado significativas, a pesar de que era esperable, al menos, que el aumento de precio de las carnes desde 2007 hubiese estimulado la producción. Respecto a la producción láctea, como en el caso anterior, cabe destacar que los rendimientos son los que contribuyen en mayor proporción a explicar los cambios observados en la variable dependiente. Al introducir las variables con retardos se observa que ninguna variable retardada es significativa, por lo tanto la especificación es menos útil para explicar el valor de la producción de leche de vaca, lo que resulta razonable dado el tipo de actividad productiva que estamos considerando. Al introducir las variables ficticias vemos que el modelo cambia ya que ambas resultan significativas. El valor positivo de VFP indica que en los años 2007, 2008 y 2009 el aumento medio de producción fue mayor que en los años anteriores, por lo tanto la producción se incrementó más en ese periodo por aumento del precio del producto. Por el contrario, el valor negativo de VFR indica que si se incluye el año de puesta en marcha de la Revisión Intermedia de la PAC (periodo 2006-2009) entonces el incremento de producción fue algo menor que en el resto de años, dado que el producto no se relaciona con el pago único.

\section{CONCLUSIONES}

Las recientes tensiones en el mercado de las materias primas y su extensión al de los alimentos, el nuevo papel de la agricultura como suministradora de energía, las políticas de protección ejercidas desde la PAC y la globalización de los mercados agrarios, vuelven a situar al sistema agroalimentario en el centro del debate económico mundial. En este trabajo nos cuestionamos cómo habrán condicionado los anteriores hechos la evolución de la producción en el sector agrario español. Para ello, se han planteado una serie de hipótesis sobre sus factores determinantes. En primer lugar, se considera la influencia positiva de las variables territoriales, se analiza el efecto positivo del precio del producto y el negativo del precio de un bien sustitutivo (en los casos en que sea factible la sustitución) y de los factores productivos y se propone la posible influencia de las subvenciones recibidas desde la PAC. En segundo lugar, se estiman los años de repuntes de precios (2007-2009) como factor que aumentará más la producción respecto a anteriores periodos, y los años de Revisión Intermedia (20062009) como factor de consecuencia incierta. 
Se han seleccionado las materias primas más representativas a partir del cálculo del porcentaje del valor de la producción de cada materia prima con respecto al valor de la producción total del subsector agrario al que pertenece. El Valor de la Producción Final Agraria de estas materias primas (cebada, tomate, pimiento, naranjo, peral, melocotonero, carne de porcino y leche de vaca), denominadas materias primas principales, ha sido considerado como variable a explicar. Mediante análisis multivariante se han planteado y ensayado cuatro modelos estadísticos para cada materia prima principal: modelo genérico, que no ha resultado concluyente por presentar problemas de no normalidad, homocedasticidad y menores coeficientes de determinación; logarítmico; con retardos, que aproxima la relevancia en las decisiones de producción de los precios del año anterior; y con variables ficticias, que examina las diferencias entre dos etapas (hasta 2007, cuando comienza la escalada de precios; y hasta 2006, cuando comienza a aplicarse la Revisión Intermedia).

Los resultados reflejan que, en general, los modelos logarítmico y con variables ficticias ofrecen mayores coeficientes de determinación, por lo que se consideran mejores en cuanto a capacidad explicativa. Por el contrario, el modelo con retardos resulta poco explicativo ya que en la mayor parte de los ensayos ofrece como respuesta que ninguna variable retardada es significativa. Ello refleja que los agricultores no basan sus decisiones en las cifras del año anterior. En todo caso podría ocurrir que aunque la evolución de los precios de los factores y el precio recibido por los agricultores fuese al alza, éstos no tuvieran capacidad para modificar su oferta en un periodo corto de tiempo, como por ejemplo en la producción de frutales o cárnicos, así que la producción podría depender de los precios de varios años atrás. En los modelos con una única variable explicativa relevante se obtienen coeficientes de determinación sensiblemente menores.

De la interpretación de las especificaciones analizadas pueden extraerse algunas conclusiones interesantes:

- La evidencia empírica demuestra que las variables territoriales son las que más contribuyen al aumento de la producción en los subsectores hortofrutícola y ganadero. En hortalizas (especialmente en el tomate) la superficie (SR) adquiere mayor importancia frente al rendimiento, que en ningún caso se obtiene significativo, dadas las características productivas de este sector. Por el mismo motivo el rendimiento (PP) ocupa la posición más favorable como predictor en los árboles frutales, especialmente cítricos (un frutal es más productivo por unidad de superficie que una planta). Como era de esperar, los rendimientos priman en el sector cárnico. En la producción porcina el peso de la canal (PC) es la única variable que resulta significativa, mientras que en la leche de vaca 
también es la variable rendimiento (RV) la que mayor influencia positiva ejerce sobre la producción.

- El coeficiente estimado para las variables relacionadas con el precio del producto (VIP y PS) es positivo y estadísticamente significativo excepto en frutales de fruto fresco no cítrico y leche de vaca, y resulta especialmente importante en los cereales. Es razonable que el precio percibido sea fundamental en cereales y hortalizas ya que son cultivos que pueden sustituirse con facilidad y sin incurrir en costes, pues tardan poco tiempo en dar fruto. Esto no sucede en frutales o ganadería, por lo que la variable precio, aunque significativa, queda en segundo plano debido a la más escasa movilidad de los factores productivos.

- Las transferencias directas de la PAC al productor no se relacionan con el aumento de la producción; la influencia que ejercen, cuando es significativa, es negativa, confirmando la no vinculación entre el nivel de producción y las subvenciones públicas. Se podría decir que la PAC, inmersa en un proceso de reforma sustancial, está consiguiendo desvincular sus ayudas directas de la producción.

- Con respecto al grado en que los precios de los factores (SM, GEN o PT) afectan al Valor de la Producción se pone de manifiesto su influencia poco significativa, sólo relevante en hortalizas y frutales no cítricos. Además su signo es positivo, contrario al esperado, lo que denota que el inexorable aumento de dichos precios a largo plazo, aunque ejerza un estímulo al abandono de la explotación, no lo hace con tanta intensidad como para quedar reflejado en el modelo, por lo tanto, la dinámica productiva del sector enmascara el posible abandono de la producción.

- La variable ficticia VFP ha permitido comprobar que en cereales y lácteos el incremento de la producción ha sido más intenso que en la etapa anterior. En estos casos, se confirma la hipótesis planteada. Por el contrario, en productos como los hortofrutícolas, donde se observan incrementos de precios mucho menores, no se ha detectado la misma influencia positiva. La variable ficticia VFR sólo se observa significativa en lácteos y hortícolas y con coeficientes negativos. Esto denota que el incremento del Valor de la Producción en los años de aplicación de la reforma ha sido menor que en la etapa anterior. El análisis de esta variable pone de manifiesto que el actual proceso de reforma de la PAC prácticamente no modifica las tendencias productivas, en coherencia con la evidencia aportada por la variable TP. 


\section{REFERENCIAS BIBLIOGRÁFICAS}

ANDRÉS, S. Y GARCÍA, J.M. (2009): Distribución regional del apoyo de la PAC a la agricultura española tras la Revisión Intermedia. Investigaciones Regionales, Vol.14, primavera, págs. 27-59.

ATANCE, I. Y GARCÍA, J.M. (2008): La evolución de los mercados agrícolas internacionales y su influencia en los precios de los alimentos. Boletín Económico del ICE, Vol. 2935, abril, págs. 11-22.

BCE (2011): Recent developments in food commodity prices. European Central Bank, Monthly Bulletin, January. Frankfurt. Disponible en: http://www.ecb.int/ pub/pdf/mobu/mb201101en.pdf (fecha de consulta: 21 de enero de 2011).

CAÑAS, J.A., FRESNO, R. Y DIOS, R. (1994): Funciones de producción líneales de variedades de maíz en Andalucía. Investigación Agraria, Vol. 9 (2), págs. 215-229.

CASTILLO J.S. Y CUEVA, M.C. (2006): Análisis de la convergencia en productividad agraria en las regiones europeas. Economía Agraria y Recursos Naturales, Vol. 10, págs. 89-105.

CEPAS, S. Y DIOS, R. (1999): Análisis de la función de producción agraria para distintos niveles de agregación. Estudios de Economía Aplicada, Vol. 12, págs. 17-33.

COELLI, T.J. Y RAO, D.S.P. (2005): Total factor productivity growth in agriculture: a Malmquist index analysis of 93 countries, 1980-2000. Agricultural Economics, Vol. 32, págs. 115-134.

CONNOR, D.J. Y LOOMIS, R.S. (2002): “Ecología de cultivos". Editado por Mundi-Prensa. Madrid.

EZCURRA, R., IRÁIZOZ, B., PASCUAL, P. Y RAPÚN, M. (2008): Spatial disparities in the European agriculture: a regional análisis, Applied Economics, Vol. 40 (13), págs. 1669-1684.

FAO (2011a): Global Food Price Monitor. Global information and early warning system on food and agriculture. (GIEWS). January. Roma. Disponible en: http://www.fao.org/giews/english/gfpm/index.htm (fecha de consulta: 2 de febrero de 2011).

FAO (2011b): Índice de la FAO para los precios de los alimentos. Sistema mundial de información y alerta sobre la alimentación y la agricultura (SMIA). Febrero. Roma. Disponible en: http://www.fao.org/worldfoodsituation /wfs-home/es/?no_cache=1 (fecha de consulta: 3 de febrero de 2011).

GARCÍA, J.M. (coordinador). (2006): "La reforma de la Política Agraria Común. Preguntas y respuestas en torno al futuro de la agricultura". Editado por EUMEDIA y Ministerio de Agricultura, Pesca y Alimentación. Colección Agronegocios. Madrid.

GARCÍA, J.M. Y RUESGA, S.M. (2007): “Economía Española”. Editado por Thomson Spain, Paraninfo S.A. Madrid. 
GARCÍA, J.M. Y GÓMEZ-LIMÓN, J.A. (coordinadores). (2010): "Chequeo Médico de la PAC. Y perspectivas de la Política Agraria Común tras 2013". Editado por EUMEDIA y Ministerio de Medio Ambiente, Medio Rural y Marino. Colección Agronegocios. Madrid.

GUTIÉRREZ, L. (2000): Convergence in US and EU agriculture. European Review of Agricultural Economics, Vol. 88, págs. 187-206.

HEADEY, D. AND FAN, S. (2010): Reflections on the Global Food Crisis. How Did It Happen? How Has It Hurt? And How Can We Prevent the Next One? International Food Policy Research Institute (IFPRI). Research Monograph 165. Washington. Disponible en: www.ifpri.org/sites/default/files/publications/ rr165.pdf (fecha de consulta: 21 de enero de 2011).

INFANTE, J. (2008): "Economía Española y Mundial: sector primario y política agraria". Editado por Universidad de Zaragoza. Zaragoza.

MORA, E.M. Y ROJAS, J. (2007): Los cultivos líderes de la agricultura venezolana (1984-2005). Agroalimentaria, Vol. 25, julio-diciembre, págs. 33-44.

REIG, E. Y PICAZO, A.J. (2002): "La agricultura española: crecimiento y productividad". Editado por Caja de Ahorros del Mediterráneo. Alicante.

REZITIS, A.N. (2005): Agricultural productivity across Europe and the United States of America, Applied Economics Letters, Vol. 12, págs. 443-446.

ROBERTS, T. (2002): Fertilidad del suelo, altos rendimientos y rentabilidad. Informaciones Agroeconómicas, Vol. 46, págs. 24-38.

TANGERMANN, S. (1997): An assesment of the Agenda 2000 proposals for the future of the Common Agricultural Policy. LUFPIG, Parlamento Europeo. 
